\title{
A case of amlodipine overdose: role of high dose insulin therapy
}

\begin{abstract}
Introduction: High dose insulin (HDI) therapy, defined as $>0.5$ units $/ \mathrm{kg} /$ hour, has been postulated to improve hemodynamics in both calcium channel blocker (CCB) and betablocker overdose. Proposed benefits of HDI include coronary and systemic vasodilation due to enhancement of nitric oxide synthase activity leading to improved contractility and decreased systemic vascular resistance, respectively. HDI also directly improves cardiac contractility by increasing glucose uptake and improving calcium handling within cardiac cells. We present a case of a patient with amlodipine overdose and her response to HDI therapy.
\end{abstract}

Case presentation: A 52year old female presented with amlodipine overdose after suicide attempt leading to shock. She received continuous intravenous infusion of regular insulin at a rate of $2 \mathrm{units} / \mathrm{kg} /$ hour and epinephrine titrated up to $80 \mathrm{mcg} /$ hour to maintain mean arterial pressure above 65 . After several hours, there was concern for worsening hypokalemia so insulin was suspended. Patient subsequently became more hypotensive, requiring further uptitration of epinephrine. When continuous insulin was later restarted, there was an observed immediate improvement in blood pressure. Patient was eventually weaned off of vasopressors, followed by insulin, by day 3 . She was downgraded from the ICU on day 5 .

Conclusion: In patients with $\mathrm{CCB}$ overdose, initial management should include fluid resuscitation, calcium supplementation, and early consideration of vasopressors and HDI therapy. Although there are currently no controlled human studies to evaluate the benefits of HDI therapy, this case revealed worsening hypotension upon cessation of therapy followed by rapid improvement in blood pressure when restarting insulin. Based on this observation, HDI therapy may have a prominent role in management of CCB overdose.Keywords: horticultural herbal products, consumerism factors, behaviours, healthy lifestyle, global businesses

Keywords: amlodipine, high dose insulin, hypertension, arrhythmias, insulin, cardiogenic shock

Abbreviations: HDI, high dose insulin; $\mathrm{CCB}$, calcium channel blocker; ED, emergency department

\section{Introduction}

Calcium channel blockers $(\mathrm{CCB})$ are a leading cause of mortality from drug overdose among cardiovascular medicine. ${ }^{1}$ CCBs are commonly used for treating hypertension, angina, and arrhythmias. However in an overdose, this can lead to cardiogenic shock that is difficult to treat with vasopressors alone and is especially problematic for long-acting agents such as amlodipine. Insulin is widely used in treatment of diabetes mellitus, hypertriglyceridemia, and hyperkalemia. High dose insulin (HDI) therapy, defined as $>0.5$ units/ $\mathrm{kg} / \mathrm{hr}$, has been postulated to improve hemodynamics in $\mathrm{CCB}$ poisonings given its positive inotropic and vasodilatory effects at the capillary beds leading to decreased vascular resistance and improved cardiac output.

\section{Case presentation}

A 52year old female with a history of hypertension, depression and previous suicide attempts was brought into the emergency department (ED) with acute encephalopathy. EMS found the patient on her bed with snoring respirations and was very difficult to arouse. There was a suicide note by the patient stating that she took amlodipine. There were no family members or housemates nearby, so the onset of ingestion could not be determined.
Volume 5 Issue 3 - 2017

\author{
Quang T Nguyen, ${ }^{1,3}$ Kelvin Tran,' Thinh Tang,' \\ Tarris Webber,' Paul J Kalekas ${ }^{2}$ \\ 'Resident, Department of Internal Medicine, Valley Hospital \\ Medical Center, USA \\ ${ }^{2}$ Assistant Program Director,Valley Hospital Internal Medicine \\ Residency Program, USA \\ ${ }^{3}$ Medical Director, Las Vegas Endocrinology, Clinical Associate \\ Professor,Arizona College of Osteopathic Medicine, and \\ Adjunct Associate Professor of Endocrinology, University \\ Nevada, USA
}

Correspondence: Quang T Nguyen, Medical Director, Las Vegas Endocrinology, Clinical Associate Professor, Clinical Education, Arizona College of Osteopathic Medicine, and Adjunct Associate Professor of Endocrinology, Touro, University Nevada, USA, Email qtn303@gmail.com

Received: July 21, 2017 | Published: August 18, 2017
Upon arrival in the ED, the patient was found to be hypotensive with blood pressure 68/53. The patient weighed $65.6 \mathrm{~kg}$. On exam, she appeared stuporous. There were significant snoring respirations but no other abnormal lung sounds were appreciated bilaterally. Heart auscultation was unremarkable. There were no signs of acute injury on abdominal exam. Cranial nerves 2-12 were grossly intact. She opens her eyes to her name and has spontaneous movements in all extremities but she does not follow commands. Initial laboratory studies, as shown in Table 1, revealed hypokalemia and mildly decreased $\mathrm{CO}_{2}$. The corrected calcium was normal, measured at 8.9. Urinalysis was not suggestive of a UTI and otherwise unremarkable. Her chest x-ray and computed tomography of the head on admission revealed no acute pathology.

Patient was given 3 liter boluses of normal saline and $4 \mathrm{gm}$ of calcium gluconate without adequate response to the blood pressure. She was started on intravenous regular insulin at a rate of 150 units per hour with dextrose $10 \%$ in water at rate of $50 \mathrm{ml} / \mathrm{hr}$. Epinephrine was administrated at the same time and titrated up to $80 \mathrm{mcg}$ per minute to maintain mean arterial pressure above 65 . Potassium chloride supplements were also administered to prevent hypokalemia while on insulin infusion.

Approximately eight hours after admission (day 2), the next renal panel revealed a potassium level of 2.9. Given concern for continued worsening of hypokalemia, the insulin infusion was suspended. 
Two hours later, mean arterial pressure dropped significantly requiring uptitration of epinephrine up to $90 \mathrm{mcg} /$ minute. Patient was subsequently noted to be more somnolent, and she was intubated for airway protection. hours after suspending insulin, it was then restarted at 150 units per hour with dextrose $10 \%$ at $50 \mathrm{ml} / \mathrm{hr}$. Almost immediately afterwards, there was an observed improvement in mean arterial pressure.

Table I Laboratory studies during hospital course

\begin{tabular}{lllll}
\hline Time & Day I & Day 2 & Day 3 & Day 4 \\
\hline Sodium & 138 & 136 & 133 & 134 \\
Potassium & 3 & 2.9 & 4.3 & 3.5 \\
Chloride & 108 & 109 & 107 & 104 \\
CO2 & 19 & 12 & 19 & 26 \\
Anion Gap & 11 & 15 & 7 & 4 \\
Glucose & 139 & 390 & 113 & 72 \\
BUN & 22 & 26 & 18 & 9 \\
Creatinine & 1.02 & 1.81 & 0.73 & 0.503 \\
Calcium & 8.3 & 7.5 & 7 & 6.8 \\
Albumin & 3.2 & 2.8 & 2 & 1.7 \\
Total Bilirubin & 1 & & & \\
Alkaline Phosphate & 57 & & & \\
Aspartate Aminotransferase & 12 & & & \\
Alanine Aminotransferase & 23 & & & \\
Lactic Acid & 1.5 & 7.4 & 4.1 & 1.8 \\
Creatine Kinase & 86 & & & \\
Troponin-I & $<0.015$ & & & \\
\hline
\end{tabular}

Over the next 24hours, potassium was supplemented approximately $40-50 \mathrm{mEq}$ every 4 hours along with magnesium and phosphorus. Potassium levels were well maintained and never went below 3.2. The most notable adverse effect was hypoglycemia but responding well to dextrose $50 \%$ pushes and increased dextrose $10 \%$ continuous infusion rate up to $140 \mathrm{ml}$ per hour. The patient continued to show improvement in blood pressure. By day 4, insulin and epinephrine were weaned off completely and the patient was downgraded from the ICU on day 5 .

\section{Discussion}

Two main classes of CCBs exist on the market: dihydropyridines and non-dihydropyridines (Table 2). Non-dihydropyridine CCBs have a strong affinity for the myocardium, including cardiac myocytes and pacemaker cells. They decrease myocardial contractility, decreased heart rate, and decreased conductivity making them useful for treating angina or cardiac arrhythmias. Amlodipine is a type of long-acting dihydropyridine CCB. At therapeutic doses, this class of CCBs act predominantly on L-type calcium channels on vascular smooth muscle cells with little effect on the myocardium. ${ }^{2}$ Amlodipine is commonly prescribed for hypertension given its favorable adverse effect profile compared to other antihypertensive agents. In severe dihydropyridine CCB overdose, there is less selectivity for peripheral vascular effects and cardiac adverse effects may be seen leading to refractory cardiogenic shock. CCB overdose may also inhibit L-type channels of pancreatic islet cells which decreases insulin release and can cause hyperglycemia. ${ }^{3}$ Other non-cardiovascular adverse effects following CCB overdose include confusion, agitation, altered mentation, and seizures likely due to decreased cerebral perfusion from hypotension. ${ }^{4}$
Table 2 Common CCBs used in the US

\begin{tabular}{ll}
\hline Dihydropyridine CCB & Non-dihydropyridine CCB \\
\hline Amlodipine & Verapamil \\
Clevidipine & Diltiazem \\
Nifedipine & \\
Nicardipine & \\
Nimodipine & \\
Nimodipine & \\
\hline
\end{tabular}

Numerous animal studies have shown beneficial effects of HDI therapy. In canines, HDI therapy was compared to other conventional therapies and showed significant improvement in myocardial contractility. ${ }^{5}$ Canines induced with propranolol with subsequent myocardial depression and later treated with HDI showed reversal of myocardial depression. ${ }^{6}$ In swine models, HDI and epinephrine therapy exhibited similar increases in myocardial contractility but epinephrine effects appeared to be temporary, lasting up to 90minutes despite continuous infusion up to a maximum dose of $50 \mathrm{mcg} / \mathrm{kg} /$ min. In contrast, HDI therapy maintained myocardial contractility significantly longer leading to marked increase in survival among the HDI group. ${ }^{7}$ Nifedipine overdose in pigs treated with HDI versus combination HDI and vasopressors show no significance in survivability, cardiac index, and mean arterial pressure. ${ }^{8}$

Many mechanisms have been proposed for the positive effects of HDI therapy. In contrast to vasopressors, HDI acts as a vasodilator in systemic, coronary, and pulmonary vasculature due to enhancement of endothelial nitric oxide synthase activity. Insulin enhances microvascular perfusion by increasing total blood flow to the capillary beds in the setting of cardiogenic shock. This effect decreases vascular resistance and enhances cardiac output. ${ }^{9}$ During states of significant stress, the myocardium primarily uses glucose as the preferred energy substances in contrast to fatty acids under non-stressed states. Insulin increases intracellular transport of glucose in cardiac muscle, and this is believed to contribute to increased myocardial contractility..$^{10}$ This effect has also been studied in explanted human myocardium and show increased inotropic function when exposed to insulin (Figure $1) .^{11}$

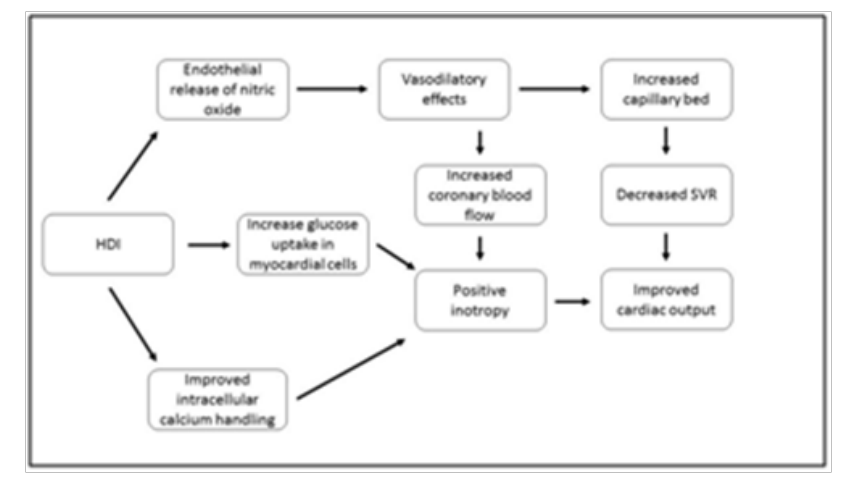

Figure I Mechanism of action of HDI therapy.

Many case reports have implicated beneficial effects of HDI therapy in calcium channel blocker and beta-blocker poisoning. There is no recommended standard dosing and rate of insulin administration but most clinicians administer an initial insulin bolus 1 unit $/ \mathrm{kg}$ followed by a $0.5-2$ units $/ \mathrm{kg} /$ hour continuous infusion. Benefits have been reported in as little as $0.015 \mathrm{units} / \mathrm{kg} /$ hour up to $22 \mathrm{units} /$ 
$\mathrm{kg}$ /hour. HDI therapy is typically started early during cardiogenic shock and at the same time as starting vasopressors., ${ }^{9,12,13}$ Once the patient is hemodynamically stable, vasopressors are weaned off first followed by HDI therapy. Cases in the literature report worse outcome and decreased survival when HDI therapy is not started early likely because of the temporarily benefits from vasopressor alone and the lack of positive inotropic effects of insulin.?

The most common adverse effect of HDI therapy is hypoglycemia. Administration of $5-10 \%$ dextrose continuous infusion and high dose dextrose pushes is given concomitantly with HDI therapy to maintain euglycemia. Consideration of glucose infusions greater than $10 \%$ through a central venous catheter may be warranted if there is concern for fluid overload. Frequent glucose checks initially every 10 minutes is recommended until glucose levels stabilize. Dextrose supplementation may need to be continued post HDI therapy due to persistent insulin concentrations. Hypokalemia is another concern of HDI therapy, as is the case with insulin infusions during treatment of diabetic ketoacidosis or hyperglycemic hyperosmolar nonketotic state. Fortunately, severe hypokalemia as defined by the presence of EKG changes is rarely seen with HDI therapy when supplemented with continuous or bolus potassium replacements. This is likely due to the shifting of potassium cations into the cell and not total body depletion of potassium. Potassium, magnesium, and phosphorus levels also should be checked frequently until levels have stabilized.

Currently, there are no clinical trials to compare the efficacy of HDI therapy alone versus vasopressor therapy alone or vasopressor and HDI therapy. Levine et al. evaluated 45patients overdosed on CCBs and showed good survival outcome on vasopressor therapy alone. ${ }^{14}$ However, early initiation of HDI has been implicated in many controlled animal trials and clinical case reports as beneficial therapy for CCB overdose. We presented a case of a patient who overdosed on amlodipine. Due to concerns for worsening hypokalemia, insulin therapy was held and subsequently there was an observed worsening of hemodynamic status. Upon re-initiation of insulin there was an immediate improvement in mean arterial blood pressures suggesting that HDI therapy may play a significant role in $\mathrm{CCB}$ overdose.

Initial $\mathrm{CCB}$ overdose management should include IV fluid resuscitation, consideration of intubation for airway protection if necessary, and laboratory/imaging studies to rule out other contributory diagnoses including infection and polysubstance use. Gastric lavage or activated charcoal may be considered if patients present less than 1hour post-ingestion. IV calcium is also recommended in early management for improvement in contractility and blood pressure because it is readily available and carries little risk. ${ }^{15-18}$ Vasopressor and HDI therapy should be considered early to maintain mean arterial pressures with frequent glucose and electrolyte monitoring. While our patient experienced hypoglycemia and hypokalemia, both issues were well managed with continuous dextrose infusions and conventional potassium replacement protocols. Based on this case and other case studies in the literature, HDI appears to be a promising treatment for CCB poisoning.

\section{Acknowledgments}

None.

\section{Conflicts of interest}

The authors declare that there are no conflicts of interest.

\section{References}

1. Mowry JB, Spyker DA, Brooks DE, et al. 2014 Annual Report of the American Association of Poison Control Centers' National Poison Data System (NPDS): 32 ${ }^{\text {nd }}$ Annual Report. Clinical Toxicology. 2015;53(10):962-1146.

2. Salhanick SD, Shannon MW. Management of calcium channel antagonist overdose. Drug Saf. 2003;26(2):65-79.

3. Ohta M, Nelson J, Nelson D, et al. Effect of $\mathrm{Ca}^{++}$channel blockers on energy level and stimulated insulin secretion in isolated rat islets of Langerhans. J Pharmacol Exp Ther. 1993;264:35-40.

4. Rietjens SJ, De Lange DW, Donker DW, et al. Practical recommendations for calcium channel antagonist poisoning. Neth J Med. 2016;74(2):60-67.

5. Kline JA, Lenova E, Raymond RM. Beneficial myocardial metabolic effects of insulin during verapamil toxicity in the anesthetized canine. Crit Care Med. 1995;23(7):1251-1263.

6. Krukenkamp I, Sørlie D, Silverman N, et al. Direct effect of high-dose insulin on the depressed heart after beta-blockade of ischemia. Thorac Cardiovasc Surg. 1986;34(5):305-309.

7. Holger JS, Engebretsen KM, Fritzlar SJ, et al. Insulin versus vasopressin and epinephrine to treat beta-blocker toxicity. Clinical Toxicology. 2007;45(4):396-401.

8. Engebretsen KM, Morgan MW, Stellpflug SJ, et al. Addition of phenylephrine to high-dose insulin in dihydropyridine overdose does not improve outcome. Clin Toxicol. 2010;48(8):806-812.

9. Engebrestsen KM, Kaczmarek KM, Morgan J, et al. High-dose insulin therapy in beta-blocker and calcium channel-blocker poisoning. Clinical toxicology. 2011;49(4):277-283.

10. Lheureux P, Zahir S, Gris $M$, et al. Bench-tobedside review: Hyperinsulinaemia/euglycaemia therapy in the management of overdose of calcium-channel blockers. Crit Care. 2006;10(3):212-217.

11. VonLewinski D, Bruns $\mathrm{S}$, Walther $\mathrm{S}$, et al. Insulin causes [Ca2b] idependent and $\left[\mathrm{Ca}^{2} \mathrm{p}\right] \mathrm{i}$-independent positive inotropic effects in failing human myocardium. Circulation. 2005;111(20):2588-2595.

12. Stellpflug SJ, Harris CR, Engebretsen KM, et al. Intentional overdose with cardiac arrest treated with intravenous fat emulsion and high-dose insulin. Clinical Toxicology. 2010;48(3):227-229.

13. Hasin T, Leibowitz D, Antopolsky M, et al. The use of low dose insulin in cardiogenic shock due to combined overdose of verapamil, enalapril and metoprolol. Cardiology. 2006;106(4):233-236.

14. Levine M, Curry SC, Padilla-Jones A, et al. Critical Care Management of Verapamil and Diltiazem Overdose With a Focus on Vasopressors: A 25-Year Experience at a Single Center. Annals of Emergency Medicine. 2013;62(3):252-258.

15. Upreti V, Ratheesh VR, Dhull P, et al. Shock due to amlodipine overdose Indian J Crit Care Med. 2013;17(6):375-377.

16. St-Onge M, Anseeuw K, Cantrell FL, et al. Expert Concensus Recommendations for the Management of Calcium Channel Blocker Poisoning in Adults. Crit Care Med. 2017;45(3):e306-e315.

17. Von Lewinski D, Bruns $\mathrm{S}$, Walther $\mathrm{S}$, et al. Insulin causes $\left[\mathrm{Ca}^{2+}\right] \mathrm{i}-$ dependent and $\left[\mathrm{Ca}^{2+}\right] \mathrm{i}$-independent positive inotropic effects in failing human myocardium. Circulation. 2005;111(20):2588-95.

18. Brass BJ, Winchester-Penny S, Lipper BL. Massive verapamil overdose complicated by noncardiogenic pulmonary edema. Am J Emerg Med. 1996;14(5):459-461.

\section{Funding}

None. 